\title{
THEORETICAL AND EXPERIMENTAL STUDY OF THE INTERIOR BALLISTICS OF A RIFLE 7.62
}

\author{
P. O. Cronemberger, \\ E. P. Lima Júnior, \\ J. A. M. Gois, \\ and A. B. Caldeira \\ Instituto Militar de Engenharia \\ Seção de Engenharia Mecânica e de Materiais \\ CEP 22290-270, Rio de Janeiro, RJ, Brasil \\ aldelio@ime.eb.br \\ Received: October 29, 2014 \\ Revised: November 30, 2014 \\ ABSTRACT \\ This study aims to examine theoretically and experimentally the interior \\ ballistics of a rifle 7.62. Three theoretical methods are employed: the \\ Vallier-Heydenreich, which is based on empirical data tables; the lumped \\ parameters that is represented by a differential-algebraic system of \\ equations, describing the propellant combustion, the thermodynamics of the \\ gas inside the gun and the projectile dynamics; and the commercial software \\ PRODAS. The theoretical solutions furnish the pressure, the projectile \\ velocity and the projectile position inside the gun, the maximum pressure, \\ the muzzle velocity and the total time of the interior ballistics. The \\ experiments measure the pressure along of the time and the projectile \\ velocity at seven meters ahead of the barrel. The proposed lumped \\ parameter model indicates alternatives to model the energy lost and the \\ resistance pressure functions. The theoretical solutions are compared with \\ experiments. A thermodynamics analysis of the energy conversion in the \\ gun is provided. The results are analyzed and the relevance of each method \\ is highlighted. \\ Accepted: December 30, 2014 \\ Keywords: interior ballistics, rifle, Vallier-Heydenreich, lumped parameter
}

\section{NOMENCLATURE}

a pressure index of the propellant

B burning rate constant of the propellant

c co-volume

D projectile diameter

E energy

f web fraction

F impetus of the propellant

$\mathrm{k}$ form function coefficient

$\mathrm{m}$ mass

M mass of the projectile

$\mathrm{P} \quad$ gas pressure

Q heat

$\mathrm{R}$ constant of the gas

$\mathrm{V}$ velocity of the projectile

Vol volume

$\mathrm{S}$ projectile position

$\mathrm{L}$ barrel length

$\mathrm{t}$ time

$\mathrm{T}$ gas temperature

$\mathrm{z}$ form function of the propellant

$\mathrm{U}$ internal energy of the gas

W kinetic energy of the projectile

Web web thickness

\section{Greek symbols}

$\gamma \quad$ ratio of specific heats of the gas

$\lambda$ dimensionless shot travel

$\rho$ density of the propellant

$\xi \quad$ thermal efficiency

$\delta, \eta, \varphi, \psi, \Theta, \Pi, \Sigma, \mathrm{T}, \Phi \quad$ Vallier-Heydenreich functions

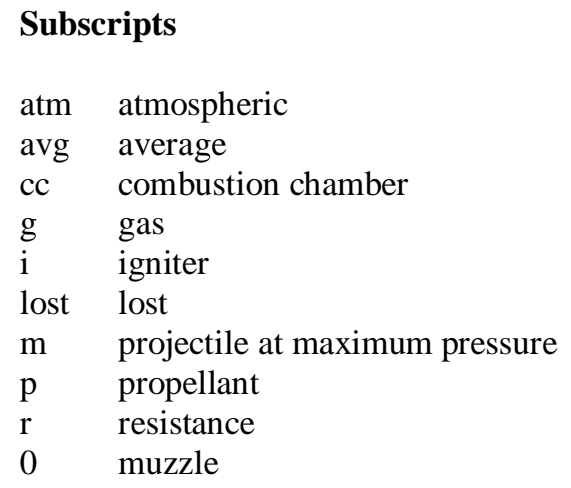

\section{INTRODUCTION}

"A conventional gun is essentially a heat engine in which the propellant contained or injected in the gun chamber is ignited and combusted, transferring its chemical energy into kinetic energy of the projectile" (Maag and Klingenberg, 1996). The interior ballistics is the science devoted to study the processes inside this heat engine. Then, "interior ballistics deals with the interaction of the gun, projectile and propelling charge before emergence of the projectile from the muzzle of the gun" (Carlucci et al., 2008).

Interior ballistics is not restricted to the gun propulsion. It is also applied to others propellant combustion systems such as: rockets, airbags, gas generators, closed vessels and primers (Corner, 1950, Lipanov, 2000, Oliveira et al., 2005, Rodrigues et al., 2006, Eisenreich et al., 2007, Seo et al., 2011).

The mathematical models of the interior ballistics are very important, since experiments are difficulty and expensive. The total elapsed time of the interior ballistics of a gun is the order of 
milliseconds. Furthermore, the pressure and the temperature inside the barrel can exceed, respectively, $400 \mathrm{MPa}$ and $3000 \mathrm{~K}$.

Interior ballistics experiments should not be intrusive and they are generally conducted by using test barrels. These experiments can measure the muzzle velocity, the maximum pressure and some of them the pressure evolution inside the barrel. Two types of pressure meters are usually employed: the piezoelectric and the crusher (Vincent, 1987). The piezoelectric measures the pressure along of the time and the crusher only measures the maximum pressure. The muzzle velocity can be measured by optical devices: high speed camera or light screens. Nevertheless, indirect measurements could be done by inverse problems techniques (Lipanov, 2000, Oliveira et al., 2005, Rodrigues et al., 2006, Arkhipov et al., 2010, 2010b). Such techniques frequently need a mathematical model of the direct problem that should be evaluated many times (Colaço et al., 2006). Then, to reduce the computational cost of the inverse problem solution, simple mathematical models of the direct problem should be used.

Moreover, for engineering purposes, simple mathematical models, mainly the lumped parameters ones, are still in use and they are relevant tools to design: propellants, ammunitions and guns ( $\mathrm{Li}$ and Zhang, 2011, 2012, Cheng and Zhang, 2012). These models are employed in optimization problems to compute the pressure, the projectile velocity and projectile position inside the gun. Consequently, despite the evolution of the interior ballistics models that take in account multiphase flows and turbulence in multidimensional domains (Krier and Summerfield, 1979, Jaramaz et al., 2011), simple mathematical models that retain the main features of the interior ballistics phenomena are still in scene.

The present work is devoted to the interior ballistics of a rifle 7.62 by using theoretical methods and experimental measurements. Three theoretical approaches are employed: the Vallier-Heydenreich method, the lumped parameters method and the commercial software PRODAS. The theoretical simulations furnish the pressure, the projectile velocity and the projectile position inside the barrel; the maximum pressure; the muzzle velocity and the total time of the interior ballistics. The experiments measure the pressure, during the interior ballistics, and the projectile velocity at seven meters ahead of the barrel. The theoretical solutions are compared with experiments. The results are analyzed and the relevance of each method is highlighted.

\section{THEORY}

\section{PRODAS simulation}

In the present work, the commercial software PRODAS (Projectile Rocket Ordnance Design and Analysis System) is used to predict the interior ballistics of a rifle 7.62 M964. This software has a library of typical weapons, ammunitions, propellants and primers. The ammunition $76280 \mathrm{M}$, the Large Rifle Primer 762 and the rifle M14 762 are chosen from this library to simulate the interior ballistics. Furthermore, Tab. 1 shows the complementary input data used in the PRODAS simulation: the barrel length, L, the volume of the combustion chamber, $\mathrm{Vol}_{\mathrm{cc}}$, the maximum pressure, $\mathrm{P}_{\mathrm{m}}$, which is the maximum gas pressure inside the gun, and the projectile velocity at the muzzle of the gun that is usually named muzzle velocity, $\mathrm{V}_{0}$.

The PRODAS simulation is first conducted by using the Empirical module. Then, these results are introduced in the Baer-Frankle module, which solves the interior ballistics by using a modified Baer and Frankle (1962) model, which is a lumped parameters model.

Table 1. PRODAS input data.

\begin{tabular}{|c|c|}
\hline PARAMETER & VALUE \\
\hline Barrel length $\left(\mathrm{S}_{\mathrm{e}}\right)$ & $533 \mathrm{~mm}$ \\
\hline $\begin{array}{c}\text { Volume of the combustion chamber } \\
\left(\mathrm{Vol}_{\mathrm{cc}}\right)\end{array}$ & $3209 \mathrm{~mm}^{3}$ \\
\hline Maximum pressure $\left(\mathrm{P}_{\mathrm{m}}\right)$ & $320.34 \mathrm{MPa}$ \\
\hline Muzzle velocity $\left(\mathrm{V}_{0}\right)$ & $840 \mathrm{~m} / \mathrm{s}$ \\
\hline
\end{tabular}

The simulation describes the thermodynamic state of the gas inside the gun and the projectile dynamics, the rotational and translational movements of the projectile, as well as the resistant pressure, which is linked to the friction force and the forces of the rifled bore on the projectile.

PRODAS considers that the rifled bore imparts spin to the projectile. The breech pressure and the pressure, acting in the base of the projectile, are computed. So, the PRODAS simulation shows a pressure gradient inside the barrel computed by empirical functions.

\section{Vallier-Heydenreich method}

The Vallier-Heydenreich method is based on empirical data tables (Oerlinkon-Buhrle AG, 1981). These data are functions of the pressure ratio, $\eta$, or of the dimensionless shot travel parameter, $\lambda$.

$$
\eta=\frac{\mathrm{P}_{\mathrm{avg}}}{\mathrm{P}_{\mathrm{m}}}
$$

The pressure ratio is defined by Eq.(1), where $P_{\text {avg }}$ is the average pressure inside the gun during the interior ballistics.

$$
\begin{gathered}
\mathrm{S}_{\mathrm{m}}=\mathrm{L} \Sigma(\eta) \\
\mathrm{t}_{\mathrm{m}}=\frac{2 \mathrm{~L} \Theta(\eta)}{\mathrm{V}_{0}} \\
\mathrm{~V}_{\mathrm{m}}=\mathrm{V}_{0} \Phi(\eta)
\end{gathered}
$$


The empirical data tables of the functions $\Sigma(\eta)$, $\Theta(\eta)$ and $\Phi(\eta)$ are employed to determine, at the moment of the maximum pressure: the projectile position, $\mathrm{S}_{\mathrm{m}}$, Eq.(2); the time, $\mathrm{t}_{\mathrm{m}}$, Eq.(3); and the velocity, $\mathrm{V}_{\mathrm{m}}$, Eq.(4).

$$
\begin{gathered}
\mathrm{t}_{0}=\frac{2 \mathrm{~L} \mathrm{~T}(\eta)}{\mathrm{v}_{0}} \\
\mathrm{P}_{0}=\mathrm{P}_{\mathrm{avg}} \Pi(\eta)
\end{gathered}
$$

The total time of the interior ballistics, $\mathrm{t}_{0}$, and muzzle pressure, $\mathrm{P}_{0}$, when the projectile exits the barrel, are also determined by the empirical data tables of the functions $T(\eta)$ and $\Pi(\eta)$, applying Eq.(5) and (6).

$$
\lambda=\frac{\mathrm{S}}{\mathrm{S}_{\mathrm{m}}}
$$

After determined the projectile position at the moment of the maximum pressure, the dimensionless shot travel parameter, $\lambda$, defined in Eq.(7), can be computed. In this equation $\mathrm{S}$ is the projectile position inside the barrel.

$$
\begin{gathered}
\mathrm{P}=\mathrm{P}_{\mathrm{m}} \psi(\lambda) \\
\mathrm{V}=\mathrm{V}_{\mathrm{m}} \varphi(\lambda) \\
\mathrm{t}=\mathrm{t}_{\mathrm{m}} \delta(\lambda)
\end{gathered}
$$

The empirical data tables of the VallierHeydenreich method for the parameter $\lambda$ furnishes the values of the functions $\psi(\lambda), \varphi(\lambda)$ and $\delta(\lambda)$, which are used to compute, respectively, the pressure, $\mathrm{P}$, the projectile velocity, $\mathrm{V}$, and the interior ballistics time, t, employing the Eq.(8) to (10).

The input data for the Vallier-Heydenreich method are shown in Tab. 2.

Table 2. Vallier-Heydenreich input data.

\begin{tabular}{|c|c|}
\hline PARAMETER & VALUE \\
\hline Barrel length $(\mathrm{L})$ & $533 \mathrm{~mm}$ \\
\hline $\begin{array}{c}\text { Maximum pressure } \\
\left(\mathrm{P}_{\mathrm{m}}\right)\end{array}$ & $320.34 \mathrm{MPa}$ \\
\hline Muzzle velocity $\left(\mathrm{V}_{0}\right)$ & $840 \mathrm{~m} / \mathrm{s}$ \\
\hline mass of propellant $\left(\mathrm{m}_{\mathrm{p}}\right)$ & $2.6762 \mathrm{~g}$ \\
\hline mass of projectile $(\mathrm{M})$ & $9.4873 \mathrm{~g}$ \\
\hline Projectile diameter $(\mathrm{D})$ & $7.62 \mathrm{~mm}$ \\
\hline
\end{tabular}

From these data in Tab. 2 is possible to determine the average pressure applying Equation bellow (Oerlinkon-Buhrle AG, 1981).

$$
P_{\text {avg }}=2 \frac{M+0.5 m_{p}}{L \pi D^{2}} V_{0}^{2}
$$

Equation (11) reveals that the work done by the gas on the projectile is equal to the kinetic energy of the projectile plus an approximation of the kinetic energy of the gas in the Vallier-Heydenreich method, considering that all of the propellant is burned.

Thus, Eq.(1) to (10) can be used to compute the main variables of the interior ballistics: $\mathrm{P}, \mathrm{V}, \mathrm{S}$ and $\mathrm{t}$.

The Vallier-Heydenreich method is a useful tool to determine, quickly and approximately, the interior ballistics of a gun, complementing experimental data. Then, if the maximum pressure and the muzzle velocity are measured, the interior ballistics can be described by the Vallier-Heydenreich method. The other hand, the initial moments of the interior ballistics cannot be evaluated by this method, because the minor $\lambda$ available is equal to 0.25 . Consequently, information about the igniter (or primer) is not required.

\section{Lumped parameters method}

The Lumped parameters method (Farrar and Leeming, 1983) is based on the projectile dynamics and on the thermodynamics of the gas provided by the propellant burning. The propellant burning follows the Robert's law

$$
\frac{\mathrm{df}}{\mathrm{dt}}=-\frac{\mathrm{B}}{\mathrm{Web}} \mathrm{P}^{\mathrm{a}}
$$

where $\mathrm{f}$ is the web fraction, $\mathrm{B}$ is the burning rate constant, $\mathrm{a}$ is the pressure index and Web is the web thickness.

In other words, the web fraction is the linear fraction of unburned propellant. It obeys the Piobert's law, imposing that the combustion proceeds in a direction normal to the unburned propellant surface.

The form function, $\mathrm{z}$, and the form function coefficient, $\mathrm{k}$, introduces the information about the geometry of the propellant. Physically, the form function is the volumetric fraction of the burned propellant.

$$
z=(1-f)(1+k f)
$$

Then, the Eq.(14) evaluates mass of the gas provided by the propellant combustion.

$$
\mathrm{m}_{\mathrm{g}}=\mathrm{m}_{\mathrm{p}} \mathrm{z}
$$

The Nobel-Abel equation of state is adopted to take in account the coalescence of the gas molecules under high pressure. In Eq.(15), $\mathrm{Vol}_{\mathrm{g}}, \mathrm{R}, \mathrm{m}_{\mathrm{g}}$ and $\mathrm{T}$ are, respectively, the volume, the constant, the mass and the absolute temperature of the gas and $\mathrm{c}$ is the co-volume.

$$
\mathrm{P}\left(\mathrm{Vol}_{\mathrm{g}}-\mathrm{m}_{\mathrm{g}} \mathrm{c}\right)=\mathrm{m}_{\mathrm{g}} \mathrm{R} \mathrm{T}
$$


The $1^{\text {st }}$ law of the thermodynamics describes the energy conversion in the gun. The energy furnished by the propellant combustion and by the igniter, Q, is converted into the kinetic energy of the projectile, $\mathrm{W}$, into the variation of the internal energy of the gas, $\Delta \mathrm{U}$, and into energy lost, $\mathrm{E}_{\text {lost }}$.

$$
\mathrm{Q}=\Delta \mathrm{U}+\mathrm{W}+\mathrm{E}_{\text {lost }}
$$

The terms of Eq.(16) are expressed in Eq.(17) to (20). In these equations, $F$ is the impetus of the propellant, $\gamma$ is the ratio of specific heats of the gas and $E_{i}$ is the energy of the igniter. The definition of the impetus of the propellant establishes that it is equal to the product of the constant of the gas by the adiabatic flame temperature of the propellant burning. Thus, it is related to the maximum energy that could be furnished by the propellant combustion.

$$
\begin{gathered}
\mathrm{Q}=\frac{\mathrm{m}_{\mathrm{g}} \mathrm{F}}{\gamma-1}+\mathrm{E}_{\mathrm{i}} \\
\mathrm{W}=0.5 \mathrm{M} \mathrm{V}^{2} \\
\Delta \mathrm{U}=\frac{\mathrm{P}}{\gamma-1}\left(\mathrm{Vol}_{\mathrm{g}}-\mathrm{m}_{\mathrm{g}} \mathrm{c}\right) \\
\mathrm{E}_{\text {lost }}=0.26 \frac{\mathrm{m}_{\mathrm{g}} \mathrm{F}}{\gamma-1}
\end{gathered}
$$

The energy lost is usually a difficult term to be modeled. In the present work, it is considered that $26 \%$ of the energy supplied by the propellant combustion is lost. This value is based in the approximate distribution of liberate energy during the interior ballistics (Farrar and Leeming, 1983).

$$
\mathrm{Vol}_{\mathrm{g}}=\mathrm{Vol}_{\mathrm{cc}}-\frac{\mathrm{m}_{\mathrm{p}}}{\rho}+\pi \frac{\mathrm{D}^{2}}{4} \mathrm{~S}+\frac{\mathrm{m}_{\mathrm{g}}}{\rho}
$$

To evaluate the volume of gas, it is necessary to consider the initial volume of gas in the combustion chamber, the projectile movement and the propellant burning. The volume of gas is represented in Eq.(21), where $\rho$ is the density of the propellant.

$$
P=\frac{(\gamma-1)\left(Q-W-E_{\text {lost }}\right)}{\operatorname{Vol}_{g}-m_{g} c}
$$

The pressure is written in Eq.(22), combining Eq.(16) and Eq.(19).

$$
\mathrm{M} \frac{\mathrm{dV}}{\mathrm{dt}}=\pi \frac{\mathrm{D}^{2}}{4}\left(\mathrm{P}-\mathrm{P}_{\mathrm{atm}}-\mathrm{P}_{\mathrm{r}}\right)
$$

The translational dynamics of the projectile is described by the Newton's $2^{\text {nd }}$ law, Eq.(23), considering that the pressure, $\mathrm{P}$, acts behind the projectile and the atmospheric pressure, $\mathrm{P}_{\mathrm{atm}}$, acts ahead of the projectile. Moreover, there is a resistance pressure, $\mathrm{P}_{\mathrm{r}}$, representing the forces of the gun structure on the projectile.

Even as the energy lost, the resistance pressure is also difficult to be modeled. In this model, it is considered equal to 5.9 $\mathrm{MPa}$. This value was chosen with basis in the minimum value of the resistance pressure used in PRODAS simulation.

$$
\frac{\mathrm{dS}}{\mathrm{dt}}=\mathrm{V}
$$

The projectile position is obtained by the velocity definition, Eq.(24).

The Runge-Kutta fourth order method is implemented to solve the system of Eqs.(12) to (24), by using a code in Scilab. The initial conditions of the problem are $\mathrm{f}=1, \mathrm{v}=0$ and $\mathrm{S}=0$. The initial pressure is equal to the atmospheric pressure and the initial volume fraction of the burned propellant is null. The input data of the lumped parameters method are shown in Tab. 3.

Table 3. Lumped method input data.

\begin{tabular}{|c|c|}
\hline PARAMETER & VALUE \\
\hline Projectile diameter $(\mathrm{D})$ & $7.62 \mathrm{~mm}$ \\
\hline Mass of projectile $(\mathrm{M})$ & $9.4873 \mathrm{~g}$ \\
\hline Mass of propellant $\left(\mathrm{m}_{\mathrm{p}}\right)$ & $2.6762 \mathrm{~g}$ \\
\hline Density of the propellant $(\rho)$ & $1577.8 \mathrm{~kg} / \mathrm{m}^{3}$ \\
\hline Web thickness $(\mathrm{Web})$ & $0.28 \mathrm{~mm}$ \\
\hline $\begin{array}{c}\text { Burning rate constant of the } \\
\text { propellant }(\mathrm{B})\end{array}$ & $7.8710^{-7}(\mathrm{~m} / \mathrm{s}) \mathrm{Pa}^{-\mathrm{a}}$ \\
\hline $\begin{array}{c}\text { Pressure index of the } \\
\text { propellant }(\mathrm{a})\end{array}$ & 0.69 \\
\hline Impetus of the propellant $(\mathrm{F})$ & $977.4 \mathrm{~kJ} / \mathrm{kg}$ \\
\hline $\begin{array}{c}\text { Ratio of specific heats of the } \\
\text { gas }(\gamma)\end{array}$ & 1.24 \\
\hline $\begin{array}{c}\text { Volume of the combustion } \\
\text { chamber }\left(\text { Vol }_{\mathrm{cc}}\right)\end{array}$ & $3209 \mathrm{~mm}$ \\
\hline Form function coefficient $(\mathrm{k})$ & 0 \\
\hline Co-volume $(\mathrm{c})$ & $6901 \mathrm{~m}^{3} / \mathrm{kg}^{3}$ \\
\hline Igniter energy $\left(\mathrm{E}_{\mathrm{i}}\right)$ & \\
\hline
\end{tabular}

\section{EXPERIMENTS}

In this work, the interior ballistics experiments are conducted in a test barrel of the rifle 7.62 M964 with a piezoelectric pressure meter with maximum pressure equal to 4000 bar. The pressure inside the barrel is measured along of the time. The pressure meter is positioned just ahead of the cartridge case in the test barrel. The projectile velocity is measured at seven meters ahead of the barrel by a precision light screen device. This velocity is considered approximately equal to muzzle velocity. Two shot experiments are executed with ammunition 7.62. The uncertainties of the pressure meter and velocity meter are, respectively, $\pm 0.01 \mathrm{MPa}$ and $\pm 0.01 \mathrm{~m} / \mathrm{s}$. 


\section{RESULTS}

The results of the theoretical and experimental study of the interior ballistics of the rifle 7.62 M964 are present in this section.

The Fig. 1 shows the projectile velocity along of the time obtained by the PRODAS, VallierHeydenreich and lumped parameters methods, while Fig. 2 reports the projectile velocity evolution along of projectile position.

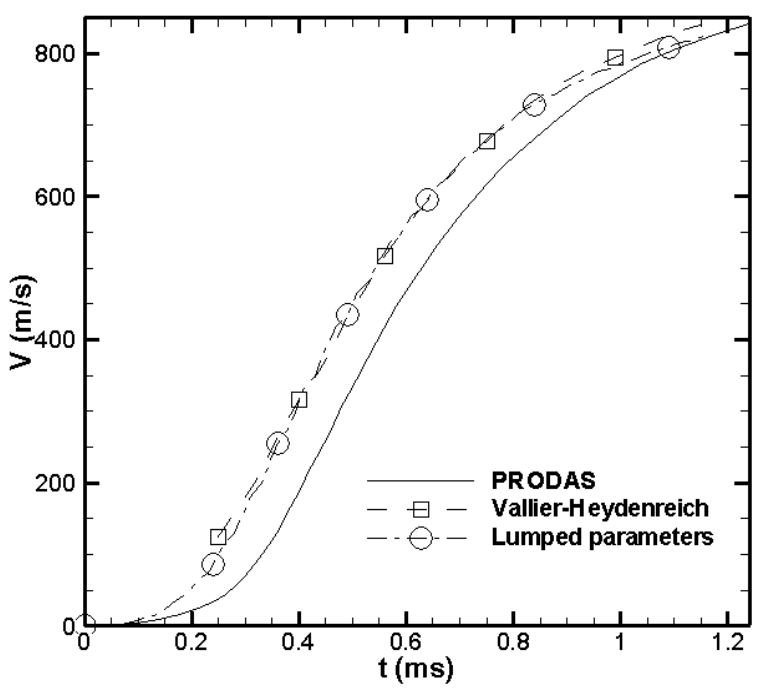

Figure 1. Projectile velocity along of the time.

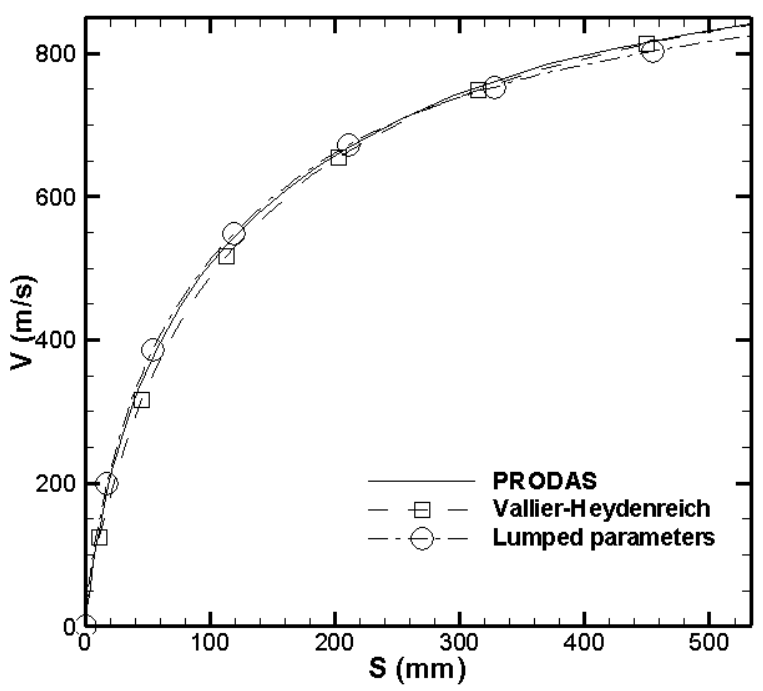

Figure 2. Projectile velocity along of the barrel.

In Fig. 1, the Vallier-Heydenreich and lumped parameters show the best match curves. Moreover, these methods predict higher velocities than PRODAS results along of the time. Otherwise, in Fig. 2 , the PRODAS and lumped parameters show the best match curves until the middle of the barrel, where Vallier-Heydenreich and PRODAS curves become closer. It is explained by the input data in the
Tab. 1 and 2, where the muzzle velocity is a parameter.

The pressure evolution along of the time obtained by the three studied theoretical methods and experiments are shown in Fig. 3. Relevant differences between the experimental and theoretical results are observed. There is a delay between theoretical and measured results. Nevertheless, before the point of maximum pressure, the slopes of experimental curves are greater than the slopes of the simulated ones. However, after the maximum pressure point, the curves show similar slopes. These differences could be explained by the experimental procedure. The pressure meter is positioned after the cartridge case and the data are recorded after the pressure exceeds $20 \mathrm{MPa}$. Therefore, measurements start after the projectile exits the cartridge case, but the simulations start before this event. Moreover, the experimental pressure is measured at a specific point in the barrel, while the pressure provided by the theoretical methods is related to the base pressure. It is important to highlight that Vallier-Heydenreich and lumped parameters methods do not consider the pressure gradient inside the gas, differing from the PRODAS model that furnishes results for the breech pressure and for the base pressure.

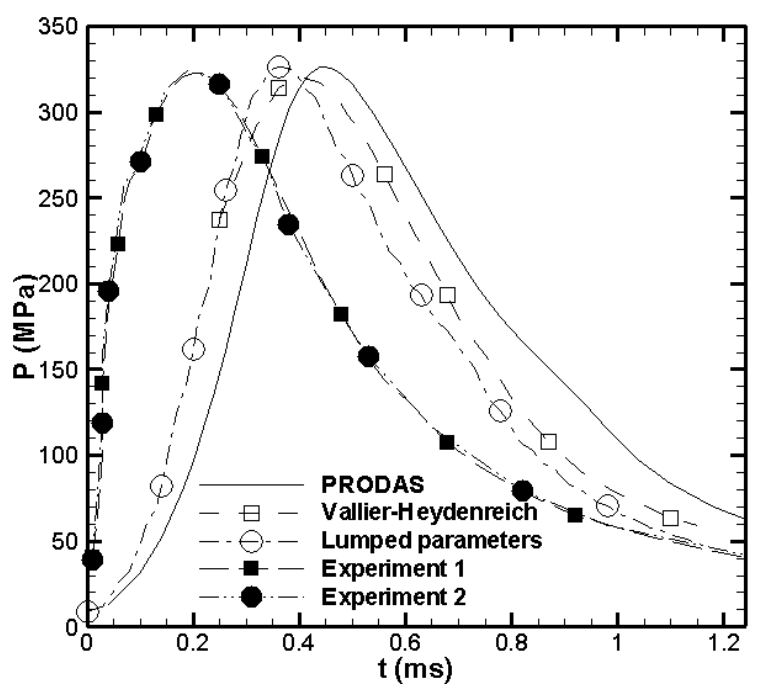

Figure 3. Pressure along of the time.

In Fig. 3, the lumped parameters results reach the maximum pressure value faster than the others theoretical results. So, in this method, the propellant burns faster. It is a consequence of the different burning law adopted in PRODAS and in lumped parameters modeling. Furthermore, the burning law parameters of the PRODAS model were not available.

The pressure evolution along of the projectile position evaluated by the theoretical methods is represented in Fig. 4. The PRODAS and the lumped parameters curves are very similar until the maximum pressure point, predicting the same 
maximum pressure at the same position. But, after this point, the lumped parameters pressure decreases faster than the others results. It is related with the fast propellant burning in the lumped parameters model. Then, in this model, the propellant is totally burned earlier.

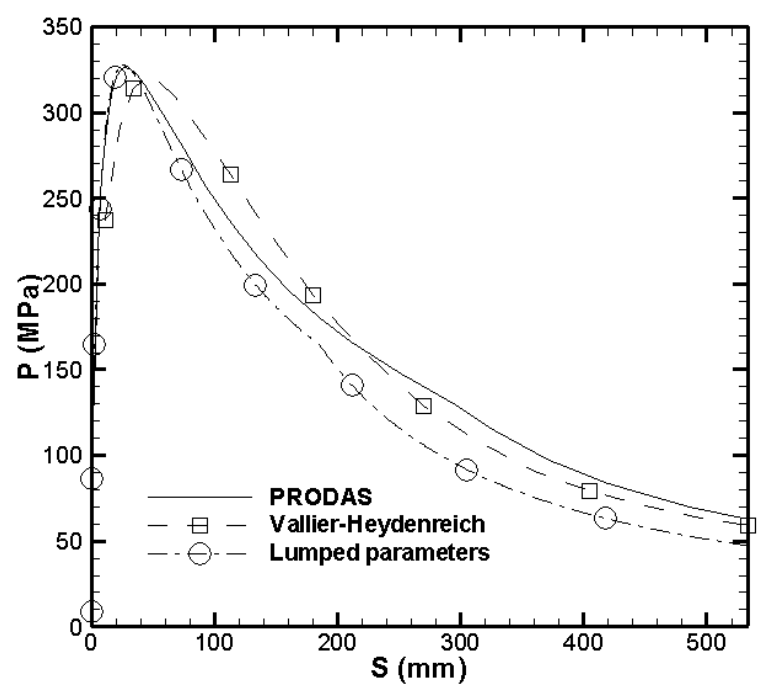

Figure 4. Pressure along of the projectile travel.

The projectile position along of the time is shown in Fig. 5. These results show that the VallierHeydenreich and the lumped parameters curves are very similar. It indicates that the total time of the interior ballistics predicted by these methods are minor than the one obtained by the PRODAS simulation. It agrees with the results shown in Fig. 1, where the velocities of the Vallier-Heydenreich and the lumped parameters are higher than PRODAS ones.

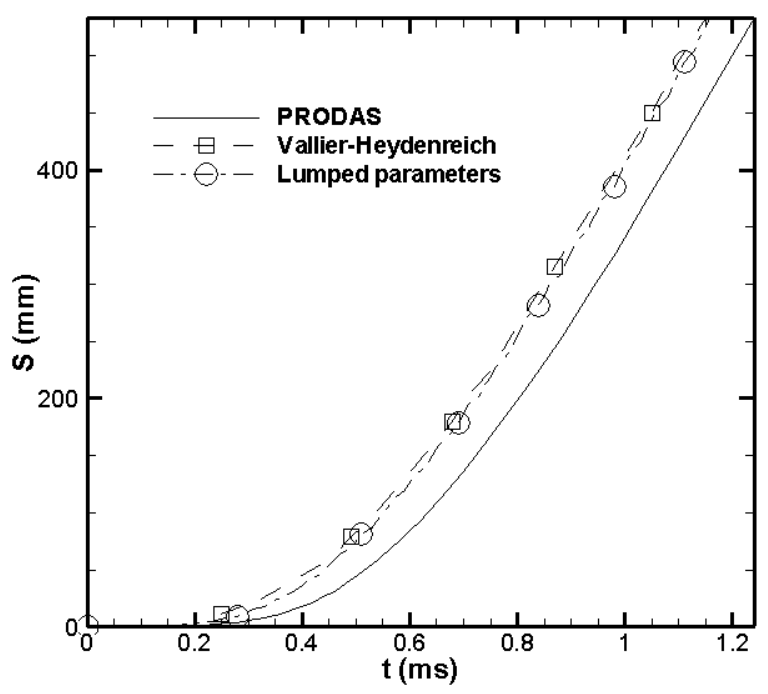

Figure 5. Projectile position along of the time.

The earlier times of the interior ballistics cannot be evaluated by the Vallier-Heydenreich method.
Thus, in Fig. 1 to 5, this period is not represented in the Vallier-Heydenreich curves.

Table 4 summarizes the main interior ballistics results obtained by studied methods and experiments. Comparing experimental results with theoretical ones, it is shown that the lumped parameters and the PRODAS simulation predicted the best results for the maximum pressure and the Vallier-Heydenreich states the best one for the muzzle velocity. However, among the studied theoretical procedures, the lumped parameters method is the only one that does not require the muzzle velocity or the maximum pressure to start the simulation. In addition, the discrepancies between theoretical and experimental results are approximately $1 \%$.

Table 4. Summary of the interior ballistics data.

\begin{tabular}{|c|c|c|c|c|}
\hline Method & $\begin{array}{c}\mathrm{P}_{\mathrm{m}} \\
(\mathrm{MPa})\end{array}$ & $\begin{array}{c}\mathrm{P}_{0} \\
(\mathrm{MPa})\end{array}$ & $\begin{array}{c}\mathrm{t}_{0} \\
(\mathrm{~ms})\end{array}$ & $\begin{array}{c}\mathrm{V}_{0} \\
(\mathrm{~m} / \mathrm{s})\end{array}$ \\
\hline PRODAS & 326 & 63 & 1.24 & 842 \\
\hline $\begin{array}{c}\text { Vallier- } \\
\text { Heydenreich }\end{array}$ & 320 & 59 & 1.15 & 840 \\
\hline $\begin{array}{c}\text { Lumped } \\
\text { parameters }\end{array}$ & 326 & 48 & 1.16 & 825 \\
\hline $\begin{array}{c}\text { Experiments } \\
\text { average }\end{array}$ & 324 & - & - & 834 \\
\hline
\end{tabular}

The muzzle pressure and the total time of the interior ballistics were not presented in Tab 4, because they were not measured. Although, the Fig. 3 indicates that the experimental muzzle pressure is closer to the lumped parameter muzzle pressure than the ones computed by the others theoretical methods.

The low muzzle pressure computed by the lumped parameters model is a consequence of the fast propellant burning process. Thus, a long period for the gas expansion without any energy input is simulated, decreasing the pressure to improve the projectile velocity.

In absence of an experimental data, PRODAS simulation can be considered the reference result for $\mathrm{t}_{0}$. The discrepancies between the theoretical results reach $7 \%$. The greatest total time for the interior ballistics is evaluated by the PRODAS. It is attributed to the burning law and to the resistance pressure. In relation to the lumped parameters model, the propellant burns slowly and the average resistance pressure is higher in the PRODAS simulation, because it considers the forces of the rifled bore on the projectile. It also explains the lower values of the PRODAS curves in Fig. 1 and 5.

$$
\xi=\frac{\mathrm{W}}{\mathrm{Q}}
$$

Since, the gun is a heat engine, the thermal efficiency could be evaluated by Eq.(25).

According with the data in Tab. 4, the thermal efficiency of the gun varies from $29 \%$ to $31 \%$, which 
agrees with the typical values of the $30 \%$ and $32 \%$ reported by Farrar and Leeming (1983).

The energy conversion in this gun, computed by the lumped parameters method, is shown in Fig. 6. These results report the energy evolution along of the projectile position. The curves are the energy furnished by the propellant combustion and by the igniter, $\mathrm{Q}$, the kinetic energy of the projectile, $\mathrm{W}$, the variation of the internal energy of the gas, $\Delta \mathrm{U}$, and energy lost, $\mathrm{E}_{\text {lost }}$.

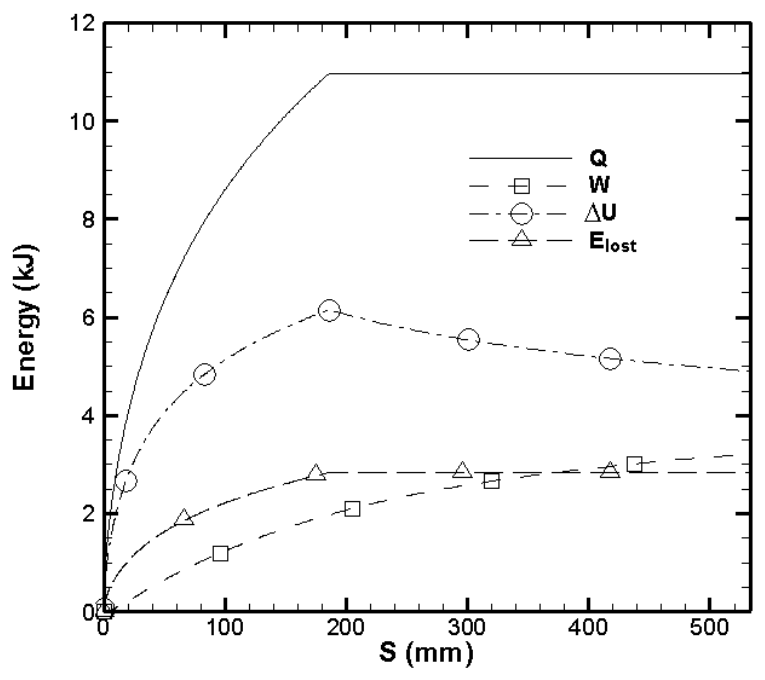

Figure 6. Energy conversion in the gun (lumped parameters results).

The heat that enters in the system reaches a maximum in the vicinity of the $180 \mathrm{~mm}$ of the projectile travel. This is the all burnt point where all of the propellant was consumed and there is no more heat from the propellant combustion to be supplied to the propulsion system. This maximum value of the energy is represented by the horizontal line in the curve Q in Fig. 6. After the all burnt point, the gas is submitted to a polytrophic expansion, reducing the internal energy to increase the projectile velocity. The all burnt point can also be observed in the lumped parameters results in Fig. 4, where the derivative of the pressure curve is discontinuous.

In Fig. 6, the energy lost increases by the influence of the resistance pressure in the initial projectile travel, but mainly by the heat transfer to the gun structure and to the projectile. Usually, the energy lost by heat transfer corresponds to $20 \%$ of the propellant energy, while the energy lost by friction represents $3 \%$ of the propellant energy (Farrar and Leeming, 1983). Thus, the energy lost curve has a maximum plateau, showing that no more energy is lost. In fact, the friction effect dissipates energy during all of the projectile travel, but this energy is very small in relation to the amount of total energy in the system. Furthermore, the heat transfer decreases along of the time, since the gun and projectile became warm and, after the all burnt point, the heat source furnished by the combustion process is no more present. Consequently, the proposed energy lost modeling implies that the gas is submitted to an adiabatic expansion, which is a special case of the polytrophic expansion.

\section{CONCLUSION}

This work studied the interior ballistics of a rifle 7.62 by using theoretical methods and experimental data. Three theoretical methods were used to evaluate the interior ballistics: the Vallier-Heydenreich, the lumped parameters and the PRODAS.

The theoretical and experimental results are in agreement and the discrepancies found are due to the experimental procedure adopted. In the experiments, the pressure meter is just ahead of the cartridge case and the timer starts only after the pressure reaches 20 $\mathrm{MPa}$. Moreover, the experimental pressure is measured in a fixed point of the barrel and the simulated pressures are related to a moving point on the base of the projectile.

The PRODAS simulation could be considered the reference result among the studied methods. This software has the more sophisticated method, taking in account more information about the ammunition and the gun. It considers the rotational movement of the projectile, the friction force and the forces of the rifled bore on the projectile.

The Vallier-Heydenreich method is an empirical method useful to complement interior ballistics experiments. It permits to evaluate the pressure, the projectile velocity and the projectile position along of the time. This method does not represent the initial instants of the interior ballistics. In addition, the muzzle velocity and the maximum pressure are required to start the evaluations.

The proposed lumped parameters model is based on the thermodynamics of the gas inside the gun and on the projectile dynamics. This method is important as an academic tool. The interior ballistics and thermodynamics concepts can be taught with this method.

The effects of the propellant, ammunition and gun parameters on the interior ballistics can be investigated with the lumped parameters method. This method can easily be adapted to many others problems of interior ballistics. The simplicity of this method became it useful in inverse problems and optimizations problems.

Nevertheless, the lumped parameters method compared with the experimental data, in the present work, has computed the better results for the maximum pressure and muzzle pressure.

Furthermore, among the studied theoretical procedures, the lumped parameters method is the only one that does not require information about the muzzle velocity or the maximum pressure to start the simulation.

The proposed lumped parameter method indicates simple strategies to model the energy lost 
and the resistance pressure functions. These functions and the burning law are the main differences between the PRODAS model and the proposed lumped parameters model.

The analysis of the energy conversion in the gun shows the presence of two important periods limited by the all burnt point in the interior ballistics. The first period is characterized by the presence of the propellant combustion and the second one by the absence of the solid propellant. In the second period, the gas is submitted to a polytrophic expansion, reducing the internal energy to increase the projectile velocity.

All of the studied methods have limitations, but they provide relevant engineering information. The main phenomena of interior ballistics are well represented by the studied methods. Despite the simplicity of these methods, they are still useful in engineering and in research.

\section{ACKNOWLEDGEMENTS}

The authors acknowledge the support from IME and CAEx.

\section{REFERENCES}

Arkhipov, V. A., Bondarchuk, S. S., and Korotkikh, A. G., 2010, Comparative Analysis of Methods for Measuring the Transient Burning Rate. I. Research Methods, Combustion, Explosion and Shock Waves, Vol. 46, No. 5, pp. 564-569.

Arkhipov, V. A., Bondarchuk, S. S., and Korotkikh, A. G., 2010, Comparative Analysis of Methods for Measuring the Transient Burning Rate. II. Research Results, Combustion, Explosion and Shock Waves, Vol. 46, No. 5, pp. 570-577.

Baer, P. G., and Frankle, J. M., 1962, The Simulation of Interior Ballistics of Guns by Digital Computer Program, Report No 1183, Ballistic Research Laboratories, Aberdeen Proving Ground, Maryland, USA, p. 72.

Carlucci, D. E., and Jacobson, S. S., 2008, Ballistics: theory and design of guns and ammunition, CRC Press, USA, $496 \mathrm{p}$.

Cheng, C., and Zhang, X., 2012, Interior Ballistic Charge Design Based on a Modified Particle Swarm Optimizer, Structural and Multidisciplinary Optimization, Vol. 46, No. 303-310.

Colaço, M. J., Orlande, H. R. B., and Dullikravich, G. S., 2006, Inverse and Optimization Problems in Heat Transfer, Journal of the Brazilian Society of Mechanical Sciences and Engineering, Vol. 23, No. 1, pp. 1-24.

Corner, J., 1950, Theory of the Interior Ballistics of Guns, John Wiley \& Sons.

Eisenreich, N., Neutz, J., Seiler, F., Hensel, D., Stanc, M., Tesitel, J., Price, R., Rushworth, S., Markert, F., Marcelles, I., Schwengler, P., Dyduch, Z., and Lebeck, K., 2007, Airbag for the Closing of
Pipelines on Explosions and Leakages, Journal of Loss Prevention in the Process Industries, Vol. 20, pp. 589-598.

Farrar, C. L., and Leeming, D. W., 1983, Military Ballistics: a Basic Manual, Brassey's Battlefield Weapon System \& Technology, v. X, Brassey's Defense Publisher, England.

Jaramaz, S., Mickovic, D., and Elek, P., 2011, Two-Phase Flows in Gun Barrel: Theoretical and Experimental Studies, International Journal of Multiphase Flow, Vol. 37, pp. 475-487.

Kilingenberg, G., Knochel, H., and Maag, H. -J., 1995, Gun Propulsion Concepts. Part I: Fundamentals, Propellants, Explosives, Pyrotechnics, Vol. 20, pp. 304-310.

Krier, H., and Summerfield, M., 1979, Interior Ballistics of Guns, Progress in Astronautics and Aeronautics, Vol. 66, pp. 385.

Li, K., and Zhang, X., 2011, Multi-Objective Optimization of Interior Ballistic Performance Using NSGA-II, Propellants Explosives and Pyrotechnics, Vol. 36, pp. 282-290.

Li, K., and Zhang, X., 2012, Using NSGA-II and TOPSIS Methods for Interior Ballistic Optimization Based on One-Dimensional Two-Phase Flow Model, Propellants Explosives and Pyrotechnics, Vol. 37, pp. 468-475.

Lipanov, A. M., 2000, Analytical Solution of the Inverse Problem of the Interior Ballistics Controlled Solid Rocket Motor, Combustion, Explosion, and Shock Waves, Vol. 36, No. 3, pp. 323-328.

Maag, H. -J., and Klingenberg, G., 1996, Gun Propulsion Concepts. Part II: Solid and liquid Propellants, Propellants, Explosives, Pyrotechnics, Vol. 21, pp. 1-7.

Oerlinkon-Buhrle A. G., 1981, Oerlinkon Pocket-Book, $2^{\text {nd }}$ Edition, Oerlinkon-Buhrle AG, Zurich, Switzerland.

Oliveira, J. L. S. P., Filho, A. A. M. F., Platt, G. M., and Peixoto, F. C., 2005, Estimation of Ballistics Parameters of Gun Propellant Through Closed Vessel Experiment Modeling, Thermal Engineering, Vol. 4, No. 1, pp. 50-55.

Rodrigues, R. L. B., Castier, M., and Peixoto, F. C., 2006, Closed Vessel Experiment Modelling and Ballistic Parameter Estimation of Gun Propellants for Lifetime Prediction, Latin American Applied Research, Vol. 36, pp. 229-233.

Seo, Y. -D, Chung, S. H., and Yoh, J. J., 2011, Automotive Airbag Inflator Analysis Using the Measured Properties of Modern Propellants, Fuel, Vol. 90, pp. 1395-1401.

Vincent, R., 1987, Textbook of Ballistics and Gunnery, Vol. 1, Her Majesty's Stationary Office, London, England. 